\title{
Empowering Leadership als Erfolgsfaktor in der Innovation
}

\author{
Steffen H. Schröder ${ }^{1}$. Urs Baldegger ${ }^{1} \cdot$ Kilian Klösel $^{2}$
}

Received: 8 September 2020 / Accepted: 26 December 2020 / Published online: 4 June 2021

(c) The Author(s) 2021

\begin{abstract}
Abstrakt
Das Ziel dieser Studie ist es, den Effekt von Führung als Erfolgsfaktor für die Innovationsleistung zu untersuchen. Die Innovationsleistung wird unterschieden in Produktentwicklung und Produktinnovation. Aus dem Literaturüberblick geht hervor, dass transformationale Führung positive Auswirkungen auf Innovation und Kreativität hat, während transaktionale Führung auf das Erreichen von Zielen und den Erfolg neuer Produkte ausgerichtet ist. Der passiv-vermeidenden Führung wird häufig ein negativer Effekt zugeschrieben, während Empowering Leadership positive Auswirkungen aufweist. Basierend auf einer qualitativen Studie zeigen wir die Bedeutung der unterschiedlichen Führungsverhalten und belegen diese anschliessend empirisch mit Hilfe hierarchischer Regressionen. Die unabhängigen Variablen des passiv-vermeidenden, des transaktionalen, des transformationalen und des Empowering Leaderships sowie die abhängigen Variablen der Produktentwicklung und der Produktinnovation werden bei 371 Mitarbeitenden aus Liechtenstein, Schweiz, Deutschland und Österreich erhoben. Die Ergebnisse belegen negative Effekte des passiv-vermeidenden Leadership auf beide abhängigen Variablen, wohingegen die transaktionale Führung positive Effekte auf den Produktentwicklungserfolg, jedoch negative auf die Produktinnovation hat. Transformationale Führung zeigt, dem entgegengesetzt, positiven Einfluss auf die Produktinnovation und negativen Einfluss auf den Produktentwicklungserfolg. Wir argumentieren daher, dass durch den gemeinsamen Einsatz beider Führungsverhalten in Kombination mit Empowering Leadership sowohl der Produktentwicklungserfolg als auch die Produktinnovation gesteigert werden kann.
\end{abstract}

Keywords Empowerment · Transformational $\cdot$ Transaktional $\cdot$ Produktinnovation $\cdot$ Produktentwicklung

\section{Einführung}

Führende im Innovationsbereich werden häufig als weniger effektiv wahrgenommen als jene aus Nicht-Innovationsbereichen. Dies betrifft ihre Führungskompetenzen und das Führungsverhalten, nicht jedoch ihre Fachkompetenzen technischer oder innovativer Natur (Gritzo et al. 2017). Der Erfolg von Produktentwicklungen und Produktinnovationen etablierter Unternehmen ist, wie wissenschaftliche Studien belegen, massgeblich vom Leadership in den Innovations einheiten der Unternehmen abhängig (Junquera and del Brío 2017; Paulsen et al. 2013; Stoker et al. 2001; Zuraik

\footnotetext{
Urs Baldegger

urs.baldegger@uni.li

1 Universität Liechtenstein, Fürst Franz Josef Strasse, 9490 Vaduz, Liechtenstein

2 School of Management Fribourg (HEG-FR), University of Applied Sciences and Arts Western Switzerland (HES-SO), Fribourg, Switzerland
}

and Kelly 2019). Die Forschung thematisiert nur am Rande die Fragestellung, wie sich Führende im Innovationsbereich effektiv verhalten sollen und wie sie den Erfolg von Entwicklungsprozessen beeinflussen können.

Doch was ist unter Erfolg im Innovationsbereich zu verstehen? Die Entwicklung von neuen oder die Verbesserung von bestehenden Produkten hat zumeist zwei Ausprägungen. Diese sind zum einen betriebswirtschaftliche Kennzahlen wie der gewonnene Marktanteil, der Return on Investment oder der kommerzielle Erfolg, aber auch die Einhaltung des Projektbudgets und der Projektzeit, die technische Leistung oder die Qualität der neuen Produkte (Ahmad et al. 2013). Diese Faktoren können mit dem Sammelbegriff des Produktenwicklungserfolgs zusammengefasst werden. Die zweite Erfolgsperspektive, auf welche sich die Literatur im Innovationsbereich vielfach bezieht, ist die Produktinnovation. Diese ist geprägt durch die Innovationskraft der Produkte, die Anzahl Innovationen oder neuen Ideen, die Wirkung für die Marke und die Neuheit der Produkte am Markt (Lovelace et al. 2001). Beim Erfolg im Innovationsbereich kann somit zwischen 
Produktentwicklung und Produktinnovation unterschieden werden.

Der Erfolg im Innovationsbereich ist wesentlich vom Leadership abhängig. Leadership kann als interpersoneller Beeinflussungsprozess definiert werden (Yukl and Gardner 2020). Konkret lassen sich verschiedene Führungsverhalten nach dem Grad der Partizipation der Mitarbeitenden, der Interaktionsfrequenz oder der Interaktionsqualität unterscheiden. In den letzten Jahren hat in der Führungsforschung das Full Range Leadership Modell zunehmend an Popularität gewonnen (Avolio 2011; Bass and Riggio 2006). Dieses stellt den Führenden in den Mittelpunkt des interpersonellen Beeinflussungsprozesses und ist somit ein Führungsansatz, der die Geführten weitgehend ausblendet und als heroische Perspektive bezeichnet werden kann (Bass 1999; Furtner and Baldegger 2016). Das Full Range Leadership unterscheidet zwischen drei, aufeinander aufbauenden Formen der Führung: passiv-vermeidende Führung, transaktionale Führung und transformationale Führung. Das Empowering Leadership ergänzt das Full Range Leadership um die postheroische Perspektive. Dies bedeutet, dass nicht mehr die Führenden, sondern die Geführten im Vordergrund stehen. Beim Empowering Leadership wird die Selbstbestimmung und die Autonomie der Geführten erhöht mit dem Ziel, deren Entscheidungs- und Handlungsspielraum zu erweitern (Sims et al. 2009). Erfolgreiches Empowering Leadership setzt voraus, dass der Führende bewusst entscheidet, den Entscheidungs- und Handlungsspielraum der Geführten auszubauen (Amundsen and Martinsen 2014; Furtner et al. 2013). Empirische Belege für das Full Range Leadership im Innovationsbereich sind vielfältig und gut erforscht, jedoch für die postheroische Perspektive mit dem Empowering Leadership nur selektiv vorhanden (Zhang and Bartol 2010; Zhu et al. 2019). Diese wenigen Untersuchungen zeigen weitgehend positive Effekte auf die Kreativität sowie die Leistungs- und die Innovationsfähigkeit (Hughes et al. 2018).

Ziel dieser Untersuchung ist es, die Auswirkungen der transaktionalen Führung, der transformationalen Führung und des Empowering Leadership auf die Innovation zu untersuchen. Innovation als abhängige Variable wird aufgeteilt in Produktentwicklung und Produktinnovation. Zunächst wird der Stand der Führungsforschung in innovativen Organisationen skizziert. Anschliessend werden Interviews zum Führungsverhalten mit Leitern von Innovationseinheiten dargestellt. Daran anknüpfend wird eine quantitative Untersuchung vorgestellt, die das Verhalten der Führenden und den zugeordneten Geführten thematisiert. Schliesslich werden die Ergebnisse der Interviews und der quantitativen Untersuchung diskutiert und auf dieser Basis Empfehlungen für die Praxis formuliert.

\section{Transaktionales, Transformationales und Empowering Leadership}

Zur interpersonellen Beeinflussung wurden in der Forschung verschiedene Führungsansätze und Theorien entwickelt (Antonakis et al. 2018; Hogan and Kaiser 2005). Diese Vielfalt lässt sich durch verschiedene Kriterien systematisieren. Ein aktuelles Kriterium ist die Unterscheidung in heroische und postheroische Führungsansätze (Crevani et al. 2007; Furtner \& Baldegger 2016).

Der heroische Führungsansatz umfasst alle Führungsverhalten, welche dem Führenden eine zentrale Rolle im Führungsprozess zuschreiben. Im stark personenzentrierten Full Range Leadership wird in passiv-vermeidende, transaktionale und transformationale Führung unterschieden (Avolio 2011; Bass and Riggio 2006). Zur passiv-vermeidenden Führung haben Howell und Avolio (1993) sowie Berson und Linton (2005) negative Effekte identifiziert. In den Untersuchungen von Jung et al. (2008), Liu et al. (2011) sowie Ryan und Tipu (2013) konnten signifikant positive Effekte des transaktionalen Leadership auf die Produktentwicklung nachgewiesen werden. Bereits frühe Untersuchungen konnten positive Effekte des transformationalen Leadership auf den Erfolg in der Innovationseinheit aufzeigen (Keller 1992; Waldman and Atwater 1994). Darüber hinaus hat das transformationale Leadership signifikant positive Effekte auf die technische Produktqualität und den Projekterfolg (Keller 2006). Weitere positive Effekte durch das transformationale Leadership stammen von Kearney und Gebert (2009), Ishikawa (2012), Peltokorpi und Hasu (2014) sowie Bednall et al. (2018). Wir nehmen daher an:

H1 Transaktionales Leadership hat einen signifikant positiven Effekt auf den Produktentwicklungserfolg.

H2 Transformationales Leadership hat einen signifikant positiven Effekt auf den Produktentwicklungserfolg und die Produktinnovation.

Crevani et al. (2007) kritisieren die Fokussierung der Leadershipforschung auf die Führenden, da die Geführten zu wenig in den Interaktionsprozess integriert werden. Neue Ansätze nehmen diese Kritik auf und zeigen, dass postheroisches Leadership, hier insbesondere das Empowering Leadership, über stärkere Effekte verfügt als das heroische Leadership (Currie and Spyridonidis 2019; Hoch et al. 2010; Nicolaides et al. 2014). Empowering Leadership wurde bisher nur selektiv in empirischen Untersuchungen integriert. Zhang und Bartol (2010) berichten, dass positive Effekte von Empowering Leadership auf die Kreativität bestehen. Zhu und Chen 
(2015) konnten zeigen, dass sich Empowering Leadership durch einen positiven Effekt auf die Leistungs- und die Innovationsfähigkeit auswirkt. Schliesslich finden auch Rao Jada et al. (2019) einen positiven Effekt des Empowering Leadership auf innovatives Arbeitsverhalten. Empirische Untersuchungen mit statistisch validierten Forschungsinstrumenten auf breiter Basis stehen bisher noch aus. $\mathrm{Zu}$ diesem Schluss kommen auch Hughes et al. (2018) in einer systematischen Literaturanalyse zu Leadership, Kreativität und Innovation. In der Literaturanalyse zeigen sie, dass sich die Forschung der Vergangenheit stark auf das heroische Führungsverhalten konzentriert hat und votieren deshalb für eine stärkere Beachtung des postheroischem Leadership (Hughes et al. 2018).

Das Empowering Leadership gliedert sich in die Förderung von unabhängigem und kooperativem Handeln sowie von opportunistischem Denken (Vecchio et al. 2010). Das unabhängige Handeln und das opportunistische Denken als Subfaktoren ermöglichen den Mitarbeitenden eine höhere Autonomie. So wird auch die intrinsische Motivation gefördert (Dewett 2007; Shirahada and Niwa 2007; Williamson et al. 2013). Zudem sind Teams in Innovationseinheiten durch eine hohe Heterogenität geprägt (Cabrales et al. 2007; Kearney and Gebert 2009; Reagans et al. 2004). Das kooperative Handeln kann hier positive Effekte haben, da Teams, die verschiedene Kompetenzen vereinen und ihre Heterogenität durch kooperatives Handeln nutzen können, erfolgreicher sind als andere (Somech 2006). Weiterhin absorbiert die Kooperation Probleme, die aus der Heterogenität der Teams und der Komplexität der Aufgaben und Projekte entstehen können (Rycroft 2007). Auf Grundlage der beschriebenen Chancen und Vorteile des Empowering Leadership für Produktentwicklung und Produktinnovation werden folgende Forschungshypothesen definiert:

H3 Empowering Leadership hat einen signifikant positiven Effekt auf den Produktentwicklungerfolg und die Produktinnovation.

H4 Das Empowering Leadership erklärt einen zusätzlichen signifikanten Varianzanteil des Produktentwicklungserfolgs und der Produktinnovation.

\section{Methodik und Vorgehen}

Die Hypothesen stützen sich sowohl auf die qualitative Untersuchung, die verschiedene Expertenmeinungen und deren Erfahrung integriert, als auch auf die Forschungsliteratur. Die Interviews bilden somit die Basis der quantitativ empirischen Untersuchung. Der Fokus liegt auf der Überprüfung der Forschungshypothesen zum transaktionalen, transformationalen und Empowering Leadership und deren Auswirkung auf Produktentwicklung und Produktinnovation. Durch die Integration von Interviews in die quantitative Überprüfung können sowohl wissenschaftliche als auch praktische Implikationen abgeleitet werden, die auch für Anspruchsgruppen in der Wirtschaftspraxis von Nutzen sind (Creswell 2013; Leech and Onwuegbuzie 2009).

Zunächst werden Leitfadeninterviews mit Experten aus forschungsintensiven Industriebetrieben durchgeführt und ausgewertet. Durch diese Interviews mit dreizehn Fragen kann ein erster Eindruck über das Innovationsumfeld, die Mitarbeitenden sowie die unterschiedlichen Führungsverhalten gewonnen werden. Diese Interviews wurden mit sieben Forschungs- und Entwicklungsleitern aus namenhaften Industrieunternehmen im Rheintal (Österreich, Schweiz und Liechtenstein) geführt, die in den Branchen Befestigungstechnik, Maschinenbau, Messtechnik, Optik, Sensorentechnik und Chemie tätig sind.

Auf den qualitativen Interviews baut die quantitative Hauptuntersuchung als Multi-Ebenen-Design auf. Es werden zwei unterschiedliche Fragebögen genutzt, jeweils für den Führenden und die Mitarbeitenden, zugeordnet über einen Zugangscode. Die Mitarbeitenden beurteilen das Führungsverhalten ihrer Vorgesetzten in Form einer Fremdbeurteilung. Die Führenden schätzen darüber hinaus den Produktentwicklungserfolg und die Produktinnovation ihrer Teams als abhängige Variablen und verschiedene Kontrollvariablen ein. Die abhängigen Variablen sind für alle Mitarbeitenden im Team einer Führungskraft identisch, sodass ein solches Vorgehen als gerechtfertigt angesehen werden kann. Alle Items der Untersuchung werden auf einer fünfstufigen Likert-Skalierung ( 1 = "trifft gar nicht zu" bis $5=$ "trifft vollkommen zu") abgetragen. Nachfolgend wird die Operationalisierung der Variablen beschrieben.

Full Range Leadership Zur Erfassung des Full Range Leadership wird der MLQ $(5 \times$ short) verwendet (Bass and Avolio 1995). Auf Grund der anhaltenden Kritik an dem Instrument führen Antonakis et al. (2003) eine umfassende Studie zur Evaluierung des MLQ $(5 \times$ short $)$ durch. Dabei nutzen die Autoren zwei unabhängige Stichproben. Bei der ersten Stichprobe mit 3.368 Teilnehmern können gute FitWerte erreicht werden $(\mathrm{RMSEA}=0.05, \mathrm{CFI}=0.91)$. Auch die zweite Stichprobe mit 6.525 Teilnehmern kann diese bestätigen (RMSEA $=0.04, \mathrm{CFI}=0.90)$. Die Autoren können die Kritik am MLQ $(5 \times$ short $)$ widerlegen, indem sie die aufgezeigten Skalenprobleme mit fehlender Homogenität innerhalb der Stichproben begründen (Antonakis et al. 2003).

Empowering Leadership Verschiedene Autoren haben zwar bereits das Empowering Leadership quantitativ erhoben (Auh et al. 2014; Srivastava et al. 2006; Xue et al. 2011), jedoch konnte bisher noch kein Instrument mit mehrfach bestätigter psychometrischer Qualität entwickelt 
werden. Arnold et al. (2000) nutzen auf Grundlage dieser Problemstellung einen neuen Fragebogen zur Erfassung von Empowering Leadership, den Empowering Leadership Questionnaire (ELQ). Die Autoren bestätigen gute Fit-Werte $(\mathrm{GFI}=0.86, \mathrm{AGFI}=0.82, \mathrm{RMSR}=0.08)$ und ein Cronbachs Alpha zwischen 0.85 und 0.91 für die Faktoren (Arnold et al. 2000). In dieser Untersuchung soll der ELQ in der Version von Vecchio et al. (2010) verwendet werden. Vecchio et al. (2010) wählen aus den sechs Faktoren der Originalfassung drei Faktoren aus: das unabhängige Handeln, das opportunistische Denken sowie das kooperative Handeln. Diese umfassen zehn Items. Vecchio et al. (2010) können ein gutes Cronbachs Alpha von 0.90 für den Hauptfaktor realisieren.

Produktentwicklungserfolg Der Produktentwicklungserfolg, hier gemessen durch die New Product Development Performance, wird durch sechs Items der von Ahmad et al. (2013) entwickelten Skala erhoben. Die Autoren greifen die New Product Development Performance nach Mallick und Schroeder (2005) auf und untersuchen deren Konstruktvalidität. Sie können dabei feststellen, dass alle sechs benannten Items nur auf einen Faktor laden und die Faktorladungen zwischen 0.60 und 0.79 liegen. Zudem überprüfen die Autoren die Reliabilität mit einem Cronbachs Alpha von 0.79 (Ahmad et al. 2013).

Produktinnovation Die Produktinnovation wird innerhalb der vorliegenden Untersuchung durch eine modifizierte Version der Team Innovation Performance (TIP) von Lovelace et al. (2001) operationalisiert. Es wird ein ähnliches Design wie in der New Product Development Performance nach Ahmad et al. (2013) verwendet. Die Items der Team Innovation Performance werden entsprechend dem Design der NPDP modifiziert. Die Reliabilität kann durch ein Cronbach Alpha von 0.87 bestätigt werden.

Kontrollvariablen Bei der Führungskraft wird die Führungserfahrung (Brown and Fields 2011), das Jahr der Unternehmensgründung und somit das Unternehmensalter (Ensley et al. 2006) sowie die Größe des Unternehmens und der Innovationseinheit durch die absolute Anzahl an Mitarbeitern erhoben (Vaccaro et al. 2012). Schliesslich wird das Championing der Innovationseinheit erfasst (Kissi et al. 2013). Dieses fragt ab, in wie fern die Ideen einer Organisationseinheit auf höherer organisatorischer Ebene vertreten werden und wie gut die Organisationseinheit somit in die Geschäftsleitung eingebunden ist (Waldman and Atwater 1994).

Innerhalb der Untersuchung sind die Fokusbranchen auf die produzierende Industrie festgelegt. Bei der Datenerhebung kann ein Sample von 145 Führenden und 452 Mitarbeitenden realisiert werden. Nach Löschung von unvollständigen Daten sind 116 Führende und 371 zugehörige Mitarbeitende zu identifizieren. Weiterhin ist zu beobachten, dass die Führenden im Mittel $45.44(\mathrm{SD}=10.43)$ Jahre alt und zu 97 Prozent männlich sind sowie über 10.47
( $\mathrm{SD}=8.37)$ Jahre Führungserfahrung verfügen. Die Mitarbeitenden sind zu 85 Prozent männlich, im Mittel 36.73 $(\mathrm{SD}=9.74)$ Jahre alt und arbeiten seit $4.45(\mathrm{SD}=3.56) \mathrm{Jah}-$ ren mit ihrer Führungsperson zusammen und seit 7.13 (SD $=6.57$ ) Jahren in ihrem Unternehmen. Die untersuchten Innovationseinheiten verfügen über $136.58(\mathrm{SD}=320.65)$ Mitarbeitende und stammen aus 44 unterschiedlichen Unternehmen, welche $54.82(\mathrm{SD}=39.21)$ Jahre alt sind. Diese sind zu 46 Prozent in Deutschland, zu 35 Prozent in der Schweiz, zu 10 Prozent in Österreich sowie zu 8 Prozent in Liechtenstein ansässig und verfügen über 24,488.91 $(\mathrm{SD}=51,338.74)$ Mitarbeitende.

\section{Qualitative Ergebnisse}

Es werden zunächst die Ergebnisse der qualitativen Leitfadeninterviews dargestellt. Diese wurden mit Experten aus der produzierenden Industrie geführt, die entweder Leiter der Forschungs- und Entwicklungsabteilung oder Leiter eines Technischen Zentrums sind. Alle Unternehmen zeichnen sich durch eine hohe Forschungsintensität aus, die sich auf die Bereiche Befestigungstechnik (Interviewpartner I), Maschinenbau (Interviewpartner II), Messtechnik (Interviewpartner III), Optik (Interviewpartner IV), Sensorentechnik (Interviewpartner V und Interviewpartner VI) und Chemie (Interviewpartner VII) beziehen.

Zum Start der Interviews wird mit den Führenden darüber gesprochen, durch welche Persönlichkeitseigenschaften sich ein typischer Mitarbeiter in ihren Bereichen auszeichnet. Die Heterogenität der verschiedenen Persönlichkeitsprofile und der zwischenmenschlichen Konflikte werden als Herausforderungen von verschiedenen Führenden angesehen:

"Es ist nicht das Ziel, Homogenität in den Persönlichkeitseigenschaften zu erzielen. Stattdessen braucht es Heterogenität. Die Leute, die heterogen sind, müssen dann aber homogen zusammenarbeiten. Das ist die Aufgabe der Führungskraft." (Interviewpartner VI).

"Die meisten Probleme, die auftreten, sind nicht technischer Natur; die schwierigsten Probleme sind menschlich bedingt. Hier setzt die Führung ein." (Interviewpartner II).

Die Interviewpartner beschreiben, dass sich die Mitarbeitenden in Innovationseinheiten deutlich von anderen Mitarbeitenden im Unternehmen unterscheiden. So verfügen diese beispielsweise über einen hohen technischen Ausbildungsstand, Neugier und Offenheit für neue Erfahrungen, sind intrinsisch motiviert herausfordernde Probleme zu lösen, verfügen aber gleichzeitig nur über eine geringe Führungsmotivation:

"Das sind ja in der Regel Menschen, die eine sehr gute Ausbildung mitbringen, die sich schon bei der Studienwahl bewusst für Maschinenbau oder Elektronik entschieden. Die 
wollen wissen, was die Welt im Innersten zusammenhält. Die sind neugierig." (Interviewpartner I).

"Von den eintretenden Mitarbeitern in der Forschung und Entwicklung wollen nur 10 Prozent Projektleiter werden und Führung wahrnehmen. Der Rest möchte Technik machen." (Interviewpartner III).

Die Persönlichkeitsstruktur der Mitarbeitenden bringt besondere Herausforderungen bezüglich der Motivation der Mitarbeitenden und des Führungsverhaltens mit sich. Die Führungskräfte stellen im Interview verschiedene Bestandteile eines erfolgreichen Führungsverhaltens fest. Ein erster Bestandteil ist, den Mitarbeitern ein herausforderndes Problem zu geben, aber gleichzeitig auch die notwendigen Ressourcen und die Autonomie zur Lösung des Problems zur Verfügung zu stellen:

"Motiviert sind die Entwickler durch den Umgang mit hoch qualitativer Soft- und Hardware. Das ist sonst frustrierend, wenn sie ein Problem lösen sollen, für das sie aber nicht ausreichend ausgestattet sind." (Interviewpartner VI).

"Ein Ingenieur braucht appetitliches Futter, attraktive Aufgaben und tolles Spielzeug, um diese zu lösen. Als zweites braucht er wenig Behinderung, also Autonomie und Freiraum. Da gehören auch Ressourcenausstattungen dazu." (Interviewpartner III).

"Mitarbeiter in der Forschung und Entwicklung sind Unternehmer in einem Unternehmen. Als Führungskraft stelle ich mich vor meine Mitarbeiter und schaffe ihnen Raum für Autonomie; ich halte ihnen den Rücken frei. Ich übersetze die Fachprobleme an das Management. " (Interviewpartner VII).

Als zweiten wichtigen Bestandteil des erfolgreichen Leadership benennen die befragten Führenden die Zielsetzung gegenüber oder gemeinsam mit den Mitarbeitenden:

"Die grössten Probleme gibt es immer am Anfang des Projekts. Hier muss ich die Ziele setzen, die erreicht werden sollen. Danach gebe ich dem Mitarbeiter Freiraum, aber auch Unterstützung durch Ressourcen und Rat, um diese Ziele zu erreichen." (Interviewpartner IV).

"Zielsetzung ist absolut wichtig. Das ist wie bei einer Bergtour. Das Ziel muss attraktiv zu erreichen sein. Dazu braucht es Freiraum und Ressourcen für die Leute." (Interviewpartner V).

Freiraum und Zielsetzung sind somit zwei wichtige Bestandteile des Leadership in unternehmerischen Organisationseinheiten. Die Anwesenheit der Führenden und vor allem das Lob an die Mitarbeitenden, als dritter Teil des Führungsverhaltens, wird durch weitere Zitate der Interviews gestützt:

"Der typische Entwickler ist nur durch eins zu motivieren: Durch Lob und durch Anerkennung." (Interviewpartner IV).

"Natürlich spielt der Stolz des Mitarbeiters auf das neue Produkt eine Rolle. Das kann und muss ich nutzen." (Interviewpartner V).
Die sieben Interviewpartner nennen das Schaffen von Autonomie und Freiraum, die klare Zielsetzung sowie das Lob und die Anerkennung von Ergebnissen der Mitarbeitenden als zentrale Bestandteile des Führungsverhaltens. Die Verhaltensweisen der Zielsetzung, des Lobes und der Anerkennung sind insbesondere im Full Range Leadership wiederzufinden. Hingegen spricht die Schaffung von Autonomie und Freiraum stärker für ein postheroisches Führungsverhalten, wie das Empowering Leadership. Beide Führungsverhalten werden in der nachfolgenden quantitativen Betrachtung mit ihrem Effekt auf den Produktentwicklungserfolg untersucht.

\section{Quantitative Ergebnisse}

Zunächst wird mit Hilfe von SPSS 26 eine Faktorenanalyse zum Test der Validität sowie ein Test auf Cronbachs Alpha zur Bestimmung der Reliabilität durchgeführt (Field 2017).

Die Faktorenanalyse prüft, ob die Items, die einen Faktor oder eine Dimension messen, auch tatsächlich von den Befragten so wahrgenommen werden (Backhaus et al. 2015). Diese kann die Faktorladungen auf alle beschriebenen Faktoren des Leadership bestätigen. Hier werden, wie in Tabelle 1 aufgezeigt, Faktorladungen zwischen 0.407 und 0.906 erreicht. Weiterhin ist durch die Analyse auf Cronbachs Alpha der Richtwert von 0.70 von Nunnally (1978) erfüllt. Die Analysen auf psychometrische Qualität können somit die Validität und die Reliabilität der Messinstrumente für verwendete Variablen bestätigen.

\subsection{Mittelwerte, Standardabweichungen und Korrelationen}

Tabelle 2 zeigt die Mittelwerte, Standardabweichungen und zero-order Pearson Korrelationen (Hemphil 2003). Die Analyse der Mittelwerte zeigt, dass das Empowering Leadership über den höchsten Mittelwert mit $3.51(\mathrm{SD}=0.97)$ verfügt, gefolgt von dem transformationalen Leadership mit $3.47(\mathrm{SD}=0.75)$, dem transaktionalen Leadership mit 3.25 $(\mathrm{SD}=0.47)$ und dem passiv-vermeidenden Leadership mit $3.22(\mathrm{SD}=0.33)$. Weiterhin kann die Korrelationsanalyse aufzeigen, dass alle Führungsverhalten signifikant mit dem Produktentwicklungserfolg korrelieren $(r s=-0.16-0.46)$. Insbesondere das Empowering Leadership zeigt eine signifikante Korrelation mit dem Produktentwicklungserfolg $(r s=0.46)$. Hypothese 3 kann somit in der deskriptiven Analyse und der Korrelationsanalyse eine erste Bestätigung finden.

Um die angenommenen Effekte der Hypothese H3 und $\mathrm{H} 4 \mathrm{zu}$ überprüfen, werden multiple hierarchische Regressionsanalysen durchgeführt. Es soll hierbei der direkte positive Effekt des Empowering Leadership auf 
Abbildung 1 Effekte von Leadership auf den Produktentwicklungserfolg

\section{Abbildung 1}

Effekte von Leadership auf den Produktentwicklungserfolg

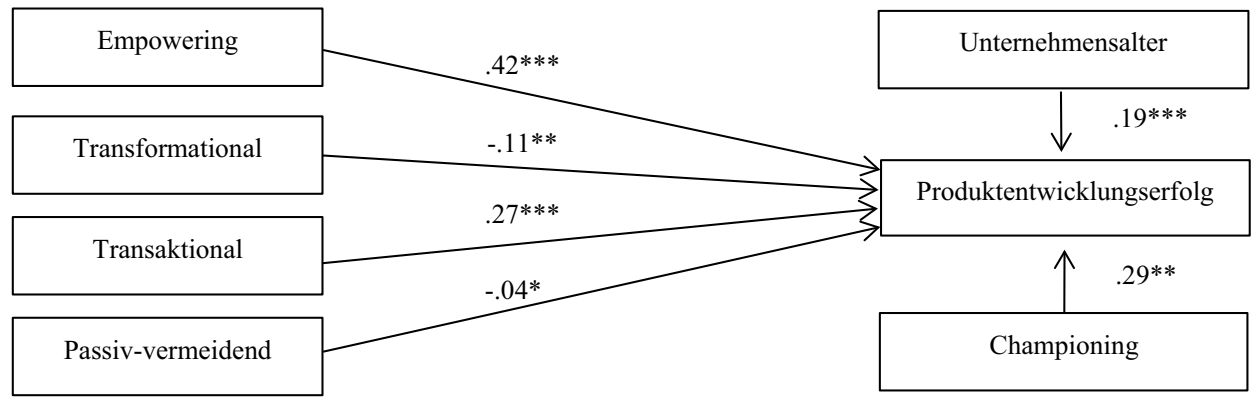

Anmerkung: $N=371 ; * \mathrm{p} \leq .05 ; * * \mathrm{p} \leq .01 ; * * * \mathrm{p} \leq .001$

Abbildung 2

Effekte von Leadership auf die Produktinnovation

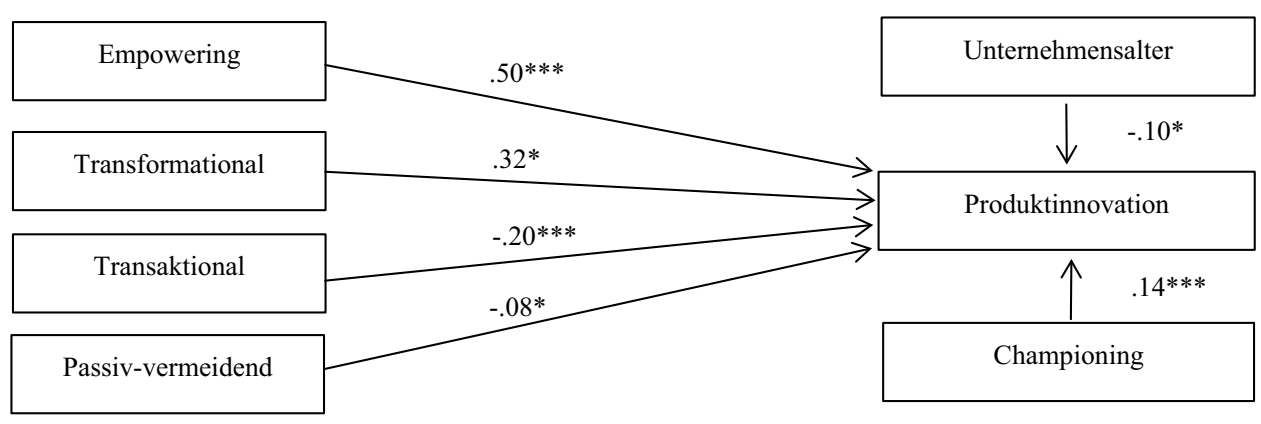

Anmerkung: $N=371 ; * \mathrm{p} \leq .05 ; * * \mathrm{p} \leq .01 ; * * * \mathrm{p} \leq .001$
Tabelle 1 Psychometrische Qualität

\begin{tabular}{lllc}
\hline Variablen: Führung und Erfolg & $\alpha$ & $\mathrm{k}$ & $\mathrm{ICC}$ \\
\hline Empowering & 0.945 & $0.614-0.819$ & 0.028 \\
Transformational & 0.935 & $0.441-0.790$ & - \\
Transaktional & 0.819 & $0.675-0.757$ & - \\
Passiv-vermeidend & 0.737 & $0.407-0.725$ & - \\
Produktentwicklungserfolg & 0.925 & $0.725-0.831$ & 0.042 \\
Produktinnovation & 0.901 & $0.728-0.906$ & 0.049 \\
\hline
\end{tabular}

Anmerkung: $N=371 ; \alpha$, Cronbachs Alpha; k, Factor loading; ICC, Intra-Class-Correlation

die Erfolgsvariablen analysiert werden, welcher über das Full Range Leadership hinausgeht. In einem ersten Modell wird das Full Range Leadership sowie das Empowering Leadership in einem Modell mit dem Produktentwicklungserfolg betrachtet. Hierzu werden auch die Kontrollvariablen in das Modell einbezogen. Das Modell erklärt dabei 31.20 Prozent der Varianz $(\mathrm{p}=0.000)$ des Produktentwicklungserfolgs. Abbildung 1 zeigt alle Effekte des Führungsverhaltens.
Diese sollen jetzt mit Hilfe multipler hierarchischer Regressionsanalysen überprüft werden (Field 2017). Es soll hierbei der direkte positive Effekt des Empowering Leaderships auf die New Product Development Performance überprüft, welcher über das Full Range Leadership hinausgeht. Das transformationale Leadership hat einen negativen Effekt $(ß=-0.11 ; p \leq 0.01)$ auf den Produktentwicklungserfolg. Das transaktionale Leadership verfügt hingegen über einen positiven Effekt ( $(=0.27 ; p \leq 0.001)$, während das passivvermeidende Leadership nicht signifikant wird. Weiterhin kann ein direkter Effekt des Empowering Leaderships auf den Produktentwicklungserfolg festgestellt werden $(\beta=0.42$; $p \leq 0.001$, Model 1), welcher höher ist als die Effekte des Full Range Leaderships. Abbildung 1 zeigt alle Effekte des Führungsverhaltens auf den Produktentwicklungserfolg.

Das transformationale Leadership hat einen negativen Effekt $(\beta=-0.11 ; p \leq 0.01)$, das transaktionale Leadership verfügt hingegen über einen positiven Effekt ( $\beta=0.27$; $p \leq 0.001$ ), während das passiv-vermeidende Leadership nicht signifikant wird. Weiterhin kann ein direkter Effekt des Empowering Leadership auf den Produktentwicklungserfolg festgestellt werden $(\beta=0.42 ; p \leq 0.001$, Model 1$)$, 
Tabelle 2 Korrelationsanalyse

\begin{tabular}{|c|c|c|c|c|c|c|c|c|}
\hline \multicolumn{2}{|r|}{$\begin{array}{l}\text { Variablen: Führung und Inno- } \\
\text { vation }\end{array}$} & \multirow{2}{*}{$\begin{array}{l}\varnothing \\
3.51\end{array}$} & \multirow{2}{*}{$\begin{array}{l}\text { SD } \\
0.97\end{array}$} & \multirow{2}{*}{$\begin{array}{l}1 \\
-\end{array}$} & \multirow[t]{2}{*}{2} & \multirow[t]{2}{*}{3} & \multirow[t]{2}{*}{4} & \multirow[t]{2}{*}{5} \\
\hline 1 & Empowering & & & & & & & \\
\hline 2 & Transformational & 3.47 & 0.75 & $0.59 * * *$ & - & & & \\
\hline 3 & Transaktional & 3.25 & 0.47 & $0.26 * * *$ & $0.27 * * *$ & - & & \\
\hline 4 & Passiv-vermeidend & 3.22 & 0.33 & $-0.21 * * *$ & 0.02 & $-0.22 * * *$ & - & \\
\hline 5 & Produktentwicklungserfolg & 3.68 & 0.93 & $0.46^{* * *}$ & $0.24 * * *$ & $0.37 * * *$ & $-0.16^{* * *}$ & - \\
\hline 6 & Produktinnovation & 3.50 & 0.86 & $0.50 * * *$ & $0.42 * * *$ & $0.24 * *$ & $-0.11 * * *$ & $0.311 * * *$ \\
\hline
\end{tabular}

Anmerkung: $N=371 ; * p \leq .05 ; * * p \leq .01 ; * * * p \leq .001$

welcher höher ist als die Effekte des Full Range Leadership. Schliesslich werden die Kontrollvariablen überprüft. Hier verfügen das Unternehmensalter $(\beta=0.19 ; p \leq 0.001)$ und das Championing $(\beta=0.29 ; p \leq 0.01)$ über einen Einfluss (Abbildung 1).

In einem zweiten Modell wird das Empowering Leadership, das transformationale Leadership, das transaktionale Leadership und das passiv-vermeidende Leadership in einem Modell mit der Produktinnovation betrachtet (Abbildung 2). Für das zweite Modell kann eine Varianzaufklärung in Höhe von 43.30 Prozent $(p=0.000)$ ausgegeben werden. Damit liegt diese über der Varianzaufklärung des Modells des Produktentwicklungserfolgs. Schliesslich postuliert Hypothese H4 eine zusätzliche Varianzaufklärung des Empowering Leadership über das Full Range Leadership hinaus. In der nachfolgenden tabellarischen Aufstellung wird die Veränderung der Varianzaufklärung zwischen den Führungsverhalten bezüglich dem Produktentwicklungserfolg und der Produktinnovation dargestellt. Es werden hier je abhängige Variable fünf verschiedene Regressionsmodelle, mit einer zunehmenden Integration von Führungsverhalten berechnet. Als Basis dienen die Kontrollvariablen und deren Aufklärung an der Varianz (Tabelle 3).

Alle identifizierten Kontrollvariablen erklären eine Varianz von $0.08(\mathrm{p}=0.000)$ bezüglich des Produktentwicklungserfolgs und $0.58(\mathrm{p}=0.001)$ der Produktinnovation. Darauf aufbauend erklärt das passiv-vermeidende Leadership eine Varianz von 13.70 Prozent $(p=0.000)$ und 19.70 Prozent $(p=0.000)$. Das transaktionale Leadership erhöht die Varianzaufklärung um 0.07 $(\mathrm{p}=0.000)$ bezüglich des Produktentwicklungserfolg und $0.01(p=0.004)$ bezüglich der Produktinnovation. Diese unterschiedliche Höhe bezüglich der Erfolgsvariablen geht einher mit den Effekten des transaktionalen Leadership auf den Produktentwicklungserfolg $(\beta=0.27, \mathrm{p}=0.000)$ und der Produktinnovation $(\beta=-0.20, p=0.000)$. Dementsprechend ist auch die Varianzaufklärung des transformationalen Leadership unterschiedlich. Während der additive Effekt des transformationalen Leadership bezüglich des Produktentwicklungserfolgs nicht bestätigt werden kann, die zusätzlich aufgeklärte Varianz beträgt $0.01(p=0.838)$, kann der Augmentationseffekt für die Produktinnovation bestätigt werden. Die aufgeklärte Varianz erhöht sich von $0.212(\mathrm{p}=0.000)$ des transaktionalen Leadership um 0.08 auf 0.29 des transformationalen Leadership $(p=0.000)$. Das Empowering Leadership erhöht die Varianzaufklärung bezüglich des Produktentwicklungserfolgs um 0.10 auf $0.31(p=0.000)$, was einer signifikanten Erhöhung entspricht. Bezüglich der Produktinnovation ist dieser Effekt noch stärker ausgeprägt. Die aufgeklärte Varianz erhöht sich hier von $0.29(\mathrm{p}=0.000)$ um 0.14 auf $0.43(\mathrm{p}=0.000)$, was nach Bühner und Ziegler (2017) einem hohen Effekt entspricht.

Die Hypothese H1 kann auf Basis der Regressionsanalyse bestätigt werden, wohingegen $\mathrm{H} 2$ nur partiell Bestätigung findet. Die Hypothesen H3 und H4 können auf Grundlage der multiplen hierarchischen Regressionen bestätigt werden, da das Empowering Leadership einen zusätzlich signifikanten Anteil an der Varianz des differenzierten Innovationserfolgs aufklärt.
Tabelle 3 Veränderung der Varianzaufklärung

\begin{tabular}{llllllll}
\hline Führungsverhalten & \multicolumn{3}{l}{ Produktenwicklungserfolg } & & \multicolumn{3}{l}{ Produktinnovation } \\
\cline { 2 - 3 } & $\mathrm{R} 2$ & $\Delta \mathrm{R} 2$ & $\mathrm{p}$ & & $\mathrm{R} 2$ & $\Delta \mathrm{R} 2$ & $\mathrm{p}$ \\
\hline Kontrollvariablen & $0.08^{* * *}$ & & 0.000 & & $0.05^{* * *}$ & & 0.001 \\
Passiv-vermeidend & $0.13^{* * *}$ & 0.05 & 0.000 & & $0.19^{* * *}$ & 0.13 & 0.000 \\
Transaktional & $0.20^{* * *}$ & 0.07 & 0.000 & & $0.21^{* *}$ & 0.01 & 0.004 \\
Transformational & 0.21 & 0.01 & 0.838 & & $0.29 * * *$ & 0.08 & 0.000 \\
Empowering & $0.31^{* * *}$ & 0.10 & 0.000 & & $0.43^{* * *}$ & 0.14 & 0.000 \\
\hline
\end{tabular}

Anmerkung: $N=371 ; * \mathrm{p}<.05 ; * * \mathrm{p}<.01 ; * * * \mathrm{p} \leq .001$ 


\section{Diskussion der Ergebnisse}

Unterschiedliche Führungsverhalten haben unterschiedliche Auswirkungen auf den Erfolg im Innovationsbereich. Dieser Zusammenhang konnte bereits hinreichend durch empirische Analysen und durch die Aufarbeitung in Meta-Analysen betrachtet werden (Burke et al. 2006; Gang Wang et al. 2011; Hughes et al. 2018; Nicolaides et al. 2014). Weniger genau untersucht wurde indes die Frage von Führungskräften, welches Führungsverhalten sie tatsächlich in ihrem Bereich einsetzen sollten, um die Produktinnovation und/oder den Produktentwicklungserfolg zu erhöhen. Diese weitgehend offene Frage wird in der vorliegenden Untersuchung zu beantworten versucht. Zur Diskussion der qualitativen und der quantitativen Ergebnisse mit der vorgestellten Literatur werden wir die Forschungshypothesen wieder aufgreifen.

In der Datenauswertung der vorliegenden Untersuchung findet sich ein positiv signifikanter Effekt des transaktionalen Leadership auf den Produktentwicklungserfolg (Hypothese 1). Die Zielorientierung des transaktionalen Leadership ist eine konsequente Ausrichtung der Mitarbeitenden auf die Produktentwicklung, wodurch ein positiver Effekt erreicht werden kann. So sprechen sich ebenfalls mehrere der Leiter von Innovationsbereichen in den Interviews für das Setzen von Zielen aus, die von ihrem Team erreicht werden sollen. Wird das transaktionale Leadership innerhalb der Innovationseinheiten betrachtet, so zeigt sich ein positiver Effekt des Führungsverhaltens auf den Erfolg (Berson and Linton 2005). Der positive Effekt auf die Produktentwicklung kann vor allem durch die Zielorientierung und die Unterstützung des Mitarbeitenden durch die Führungskraft zum Erreichen der gesetzten Ziele erklärt werden. Die abhängige Variable des Produktentwicklungserfolgs misst dabei, wie gut die vorher gesteckten Ziele bezüglich eines Entwicklungsprojekts erreicht wurden. Da die Mitarbeitenden bei der leistungsabhängigen Belohnung auf diese Ziele der Organisationseinheit oder des Entwicklungsprojektes ausgerichtet werden können, ist es mit der Unterstützung der Führungskraft zu erwarten, dass sie diese Ziele auch erreichen. Weiterhin wird durch das transaktionale Leadership die Leistungsorientierung der Mitarbeitenden aktiviert, da Leistung und Zielerreichung durch das transaktionale Leadership honoriert werden (Williamson et al. 2013; Zibarras et al. 2008).

Überraschend ist der negative Effekt des transformationalen Leadership, womit Hypothese 2 teilweise abgelehnt werden muss. Bisherige Untersuchungen weisen hingegen für beide Führungsverhalten einen positiven Effekt aus (Keller 2006; Peltokorpi and Hasu 2014). In den Interviews mit Leitern von Innovationsbereichen wurde betont, dass Lob und Anerkennung für Mitarbeitende sehr wichtig seien, da der Stolz der Mitarbeitenden auf ihre Produkte eine übergeordnete Rolle spielt. Auch deshalb ist der negative Effekt des transformationalen Leadership überraschend. Dieser Effekt kann in dem Sinne interpretiert werden, dass die individuelle Autonomie des Mitarbeitenden eingeschränkt wird, da sich die Führungskraft in den Mittelpunkt stellt. Der Mitarbeitende kann so die persönliche Leistungsorientierung nicht ausleben, da er wenig Anerkennung für Erfolge bekommt und der persönliche Freiraum durch die Führungskraft eingeschränkt wird. Die jeweils umgekehrten Effekte auf den Produktenwicklungserfolg und die Produktinnovation von transaktionalem und transformationalem Leadership verleihen der Forderung nach einem gemeinsamen Einsatz von transaktionalem und transformationalen Leadership Nachdruck. Nur so kann der Augmentationseffekt nach Bass et al. (2003) erreicht werden und sowohl der Erfolg der Produktentwicklung als auch die Produktinnovation positiv durch das Führungsverhalten beeinflusst werden.

Der signifikant positive Effekt des Empowering Leadership auf den Produktentwicklungserfolg sowie die Produktinnovation (Hypothese 3) kann durch die Datenauswertung mittels Regressionsanalyse bestätigt werden, wobei Empowering Leadership den stärksten Effekt im Vergleich mit den Führungsverhalten des Full Range Leadership zeigt. Dieser Effekt wird auch durch die qualitativen Interviews unterstützt. So beschreiben die Führungskräfte aus Entwicklungseinheiten es als essenziell, ihren Mitarbeitenden Freiraum und Autonomie einzuräumen, sie aber gleichzeitig mit den nötigen Ressourcen auszustatten und sie zu befähigen, ihre Arbeit autonom auszuführen. Alle drei Verhaltensweisen sind Elemente des Empowering Leadership, sodass ein direkter Bezug zwischen den Interviewergebnissen und dem starken positiven Effekt von Empowering Leadership hergestellt wird. Die Literatur weist bisher primär Untersuchungen auf, welche die prädiktive Erfolgswirkung des Empowering Leaderships auf die Produktinnovation bestätigen (Rao Jada et al. 2019). Gumusluoglu und Ilsev (2009) setzen das Empowering Leadership als Moderator zwischen dem transformationalen Leadership und der Innovation ein. Zhang und Bartol (2010) zeigen einen Effekt des Empowering Leadership auf die Kreativität und Zhu und Chen (2015) zeigen einen positiven Effekt auf die Leistungsfähigkeit von Teams.

Das Empowering Leadership bietet eine ganze Reihe von Argumentationsansätzen, um die starken Effekte auf den Produktentwicklungserfolg und die Produktinnovation zu erklären. Zunächst weisen die Mitarbeitenden im Innovationsbereich ein hohes Autonomiebedürfnis auf (Eisenbeiß and Boerner 2010; Hoegl and Parboteeah 2006), welches sowohl durch den Subfaktor des opportunistischen Denkens als auch des unabhängigen Handelns in der Itemallokation nach Vecchio et al. (2010) unterstützt werden 
kann. Führungskräfte motivieren hier die Mitarbeitenden Verantwortung zu übernehmen. Auch auf die intrinsische Motivation der Mitarbeitenden wird durch die Subfaktoren des Empowering Leadership eingegangen (Jordan, 2005). Weiterhin sind Teams im Innovationsbereich meist heterogen, die Aufgaben komplex und nur durch ein Team zu lösen (Kirkman and Harris 2017; Shin and Zhou 2007). Durch das kooperative Handeln nutzt die Führungskraft die Heterogenität, indem sie die Mitarbeitenden anleitet im Team zusammen zu arbeiten (Vecchio et al. 2010). Schliesslich ist das Empowering Leadership auf den Mitarbeitenden ausgerichtet, wobei sich die Führungskraft als Sparringpartner an die Seite stellt (Crevani et al. 2007; Tang et al. 2020).

Hypothese 4 bezieht sich auf die Wirkung des Empowering Leadership auf die Produktinnovation und die Produktentwicklung. Beide Vergrösserungen der Varianzaufklärung in den jeweiligen Modellen sind signifikant. Das Empowering Leadership ist auch über das Full Range Leadership hinaus ein effektives Führungsverhalten, wenn der Produktentwicklungserfolg oder die Produktinnovation beeinflusst werden sollen. Dieses Ergebnis kann bisher nicht durch Untersuchungen in der Literatur bestätigt werden, da wenige der identifizierten Untersuchungen sowohl Führungsverhalten nach dem Full Range Leadership als auch das Empowering Leadership integrieren. Dennoch ist beispielsweise die Untersuchung von Tekleab et al. (2008) aufzuführen, in welcher die Autoren positive Effekte des Empowering Leadership auf das Selbstbewusstsein von Mitarbeitern feststellen, die über das transformationale Leadership hinausgehen. Weiterhin kann auch die Aussage getroffen werden, dass das Empowering Leadership gerade in neueren Untersuchungen an Bedeutung gewonnen hat und somit in der Zukunft weiter erforscht werden sollte (Auh et al. 2014; Tekleab et al. 2008; Xue et al. 2011; Zhang and Bartol 2010). Empowering Leadership verfügt somit über hohe positive Effekte auf die Produktinnovation und den Erfolg der Produktentwicklung. Es ist somit auch nicht überraschend, dass die Varianzaufklärung durch das Empowering Leadership zunimmt. Die Integration des Empowering Leadership über das transaktionale und das transformationale Leadership hinaus bietet somit die Möglichkeit zusätzlicher Beeinflussung durch die Führungskraft. Dabei sind transaktionales Leadership, transformationales Leadership sowie Empowering Leadership nicht als substituierend, sondern als komplementär anzusehen. So schliessen weder transformationales noch transaktionales Leadership aus, das Empowering Leadership zu integrieren. Stattdessen ergänzen sich die Führungsverhalten untereinander, um einen hohen Erfolg der Produktentwicklung und Produktinnovation zu erreichen. Im Unterschied zum Full Range Leadership integriert das Empowering Leadership die Perspektive der Geführten in den Führungsprozess (Lee et al.
2019). Dies erklärt, warum Empowering Leadership den stärksten Effekt hat.

Überraschend ist, dass das passiv-vermeidende Führungsverhalten in den Regressionsanalysen negativ mit den jeweils abhängigen Variablen korreliert. Auch hier wird den Mitarbeitenden eine hohe Autonomie und Freiraum eingeräumt. So kann die vorliegende Untersuchung auch die Erkenntnisse von Wong und Giessner (2018) unterstützen. Dies führt zu der Frage nach den Unterschieden zwischen passiv-vermeidender Führung und Empowering Leadership. Die positive Wirkung des Empowering Leadership geht davon aus, dass die Führungskraft die Erwartungen der Mitarbeitenden abfragt und diesen Entscheidungs- und Handlungsspielräume gibt. Nach unserer Untersuchung sind nicht nur diese Freiräume wichtig, sondern auch die Unterstützung der Mitarbeitenden durch die Führungskraft, welche den Unterschied zwischen Empowering Leadership und der passiv-vermeidenden Führung ausmacht. Diese Argumentation wird sowohl durch die qualitativen Interviews mit Aussagen zu Unterstützung des Führenden bestätigt, vor allem aber durch die zusätzliche Varianzaufklärung des Empowering Leadership über alle anderen Führungsverhalten hinweg.

\section{Limitationen und Ausblick}

Die vorliegende Untersuchung kann zeigen, dass die strategische und die kulturelle Orientierung über signfikante Interaktionseffekte auf die Beziehung von Empowering Leadership und dem Produktentwicklungserfolg verfügen. Weiterhin kann ein signfikant positiver Effekt des Empowering Leadership auf die Erfolgsvariablen aufgezeigt werden, welcher über das Full Range Leadership hinaus geht. Unsere Studie ist nicht ohne Limitationen, welche für zukünftige Untersuchungen beachtet werden sollten. Es müssen verschiedene Limitationen bezüglich der Messinstrumente und des Untersuchungsdesigns aufgezeigt werden.

Die erste Limitation bezieht sich auf das Messinstrument des ELQ. Dieser muss in der dreifaktoriellen Anwendung nach Vecchio et al. (2010) in seiner Aussagekraft eingeschränkt werden, da eine höhere Anzahl an Items und Subfaktoren differenziertere Ergebnisse herstellen könnte (Vecchio et al. 2010). Beispielsweise kann hier die ursprüngliche Entwicklung des ELQ nach Arnold et al. (2000) oder der neue Fragebogen nach Amundsen und Martinsen (2014) als Alternative aufgezeigt werden.

Weiterhin sind auch Limitationen bezüglich des Untersuchungsdesigns auszugeben. Die vorliegende Untersuchung folgt einem Querschnittsdesign, welches auf Grund des Neuigkeitswerts der Betrachtung des Empowering Leadership in dem spezifischen Kontext explorativ ausgerichtet ist. Als Folge des explorativen Querschnittsdesigns können 
durch die Regressions- und Moderationsanalyse möglicherweise Scheinkausalitäten aufgezeigt werden, welche durch die Common Method Variance verzerrt werden (Podsakoff et al. 2003). Der Rückschluss auf bestehende Kausalität ist nur möglich, wenn ein experimentelles Design oder ein Interventionsdesign mit einer ausreichend großen Kontrollgruppe gewählt wird. Weiterhin werden die befragten Führungskräfte direkt ausgewählt, alle vorhandenen Datensätze zur Analyse verwendet und nicht zufällig aus einer Stichprobe von Führenden und Mitarbeitenden gezogen. Ferner besteht möglicherweise ein "Survivor-Bias" und eine einhergehende Erfolgsverzerrung bei teilnehmenden Führungskräften (Hair 2010).

In zukünftigen Untersuchungen ist es sinnvoll, weitere Kontrollvariablen in das Forschungsdesign zu integrieren und bedeutende Einflussvariablen, wie zum Beispiel die Marktdynamik oder die Technologieveränderungsrate, zu berücksichtigen. Diese können einen hohen Einfluss auf die Innovationskraft von Unternehmen haben. Basierend auf den qualitativen Ergebnissen erscheint es zudem lohnenswert, die Fähigkeiten der Geführten sowie deren psychologische und fachliche Reife als Antezedenzien der Selbstführung zu berücksichtigen (Cheong et al. 2019).

Eine letzte Limitation bezüglich des Untersuchungsdesigns ist in der Disaggregation der Daten zu identifizieren. So führt diese zu einem Informationsverlust, wenn zwischen den Mitarbeitenden der Multi-Ebenen Betrachtung eine hohe Varianz besteht. Obgleich keine Varianz zu ermitteln war, kann hier ein Methodenfehler nicht ausgeschlossen werden. Folgende Untersuchungen sollten das Untersuchungsdesign so gestalten, dass Leitende der Forschung \& Entwicklung befragt werden und dazu die Mitarbeitenden das jeweilige Führungsverhalten beurteilen.

\section{Zusammenfassung}

Diese Studie untersucht die Wirkung von heroischem und postheroischem Führungsverhalten im Innovationsbereich. Die Ergebnisse bestätigen, dass das Empowering Leadership einen additiven Effekt auf den Produktentwicklungserfolg aufweist, welcher über das Full Range Leadership hinausgeht. Das Empowering Leadership darf aber nicht in passiv-vermeidendes Leadership umschlagen; hier zeigen sich negative Effekte. Doch auch das transaktionale Leadership weist einen deutlich positiven Effekt auf den Produktentwicklungserfolg auf. Beide Ergebnisse werden durch die geführten Interviews mit Leitern von Innovationsbereichen verstärkt. Führenden im Innovationsbereich kann somit geraten werden, dass sie transaktional mit ihren Mitarbeitern Ziele vereinbaren, sie dann aber dann durch Empowering Leadership dazu befähigen diese zu erreichen, sie zur Kooperation mit anderen ermutigen und insbesondere
Freiraum und Autonomie geben. Die vorliegende Studie ist eine der wenigen, die den additiven Effekt des Empowering Leadership untersucht und gibt Anlass weitere Forschung in Hinblick auf die postheroische Führung im Innovationsbereich zu initiieren.

Funding Diese Studie erhielt keine spezifischen Zuschüsse von Fördereinrichtungen im öffentlichen, kommerziellen oder gemeinnützigen Sektor.

\section{Declarations}

Interessenskonflikte All authors declare that they have no conflict of interest.

Open Access Dieser Artikel wird unter der Creative Commons Namensnennung 4.0 International Lizenz veröffentlicht, welche die Nutzung, Vervielfältigung, Bearbeitung, Verbreitung und Wiedergabe in jeglichem Medium und Format erlaubt, sofern Sie den/die ursprünglichen Autor(en) und die Quelle ordnungsgemäß nennen, einen Link zur Creative Commons Lizenz beifügen und angeben, ob Änderungen vorgenommen wurden. Die in diesem Artikel enthaltenen Bilder und sonstiges Drittmaterial unterliegen ebenfalls der genannten Creative Commons Lizenz, sofern sich aus der Abbildungslegende nichts anderes ergibt. Sofern das betreffende Material nicht unter der genannten Creative Commons Lizenz steht und die betreffende Handlung nicht nach gesetzlichen Vorschriften erlaubt ist, ist für die oben aufgeführten Weiterverwendungen des Materials die Einwilligung des jeweiligen Rechteinhabers einzuholen. Weitere Details zur Lizenz entnehmen Sie bitte der Lizenzinformation auf http:// creativecommons.org/licenses/by/4.0/deed.de.

\section{References}

Ahmad S, Mallick DN, Schroeder RG (2013) New product development: impact of project characteristics and development practices on performance: NPD project characteristics and practices. J Prod Innov Manag 30(2):331-348. https://doi.org/10.1111/j. 1540-5885.2012.01002.x

Alvesson M, Spicer A (2012) A stupidity-based theory of organizations: a stupidity-based theory of organizations. J Manage Stud 49(7):1194-1220. https://doi.org/10.1111/j.1467-6486.2012. 01072.x

Amundsen S, Martinsen ØL (2014) Empowering leadership: construct clarification, conceptualization, and validation of a new scale. Leadersh Q 25(3):487-511. https://doi.org/10.1016/j.leaqua. 2013.11.009

Antonakis J, Avolio BJ, Sivasubramaniam N (2003) Context and leadership: an examination of the nine-factor full-range leadership theory using the Multifactor Leadership Questionnaire. Leadersh Q 14(3):261-295. https://doi.org/10.1016/S1048-9843(03) 00030-4

Antonakis J, Day DV (Hrsg.) (2018) The nature of leadership (3. Aufl.). SAGE Publications Inc.

Arnold J, Arad S, Rhoades J, Drasgow F (2000) The empowering leadership questionnaire: the construction and validation of a new scale for measuring leader behaviors. J Organ Behav 21:249-269. https://doi.org/10.1002/(SICI)1099-1379(200005)21:33.0.CO;2-\#

Auh S, Menguc B, Jung YS (2014) Unpacking the relationship between empowering leadership and service-oriented citizenship behaviors: a multilevel approach. J Acad Mark Sci 42(5):558-579. https://doi.org/10.1007/s11747-014-0370-0 
Avolio BJ (2011) Full range leadership development (2. Aufl.). SAGE Publications

Backhaus K, Erichson B, Weiber R (2015) Fortgeschrittene multivariate Analysemethoden: Eine anwendungsorientierte Einführung (3. Aufl.). Springer Gabler

Bass BM (1999) Two decades of research and development in transformational leadership. Eur J Work Organ Psy 8(1):9-32. https:// doi.org/10.1080/135943299398410

Bass, B.M., \& Avolio, B. J. (1995). MLQ multifactor leadership questionnaire for research. Mindgarden.

Bass BM, Riggio RE (2006) Transformational Leadership (2. Aufl.). Psychology Press. https://doi.org/10.4324/9781410617095

Bass BM, Avolio BJ, Jung DI, Berson Y (2003) Predicting unit performance by assessing transformational and transactional leadership. J Appl Psychol 88(2):207-218. https://doi.org/10.1037/ 0021-9010.88.2.207

Bednall TC, Rafferty AE, Shipton H, Sanders K, Jackson JC (2018) Innovative behaviour: how much transformational leadership do you need? Innovative behaviour. Br J Manag 29(4):796-816. https://doi.org/10.1111/1467-8551.12275

Berson Y, Linton JD (2005) An examination of the relationships between leadership style, quality, and employee satisfaction in R\&D versus administrative environments. R\&D Manag 35(1):51-60. https://doi.org/10.1111/j.1467-9310.2005.00371.x

Brown RT, Fields D (2011) Leaders engaged in self-leadership: can followers tell the difference? Leadership 7(3):275-293. https:// doi.org/10.1177/1742715011407383

Bühner M, Ziegler M (2017) Statistik für Psychologen und Sozialwissenschaftler (2. Aufl.). Pearson

Burke CS, Stagl KC, Klein C, Goodwin GF, Salas E, Halpin SM (2006) What type of leadership behaviors are functional in teams? A meta-analysis. Leadersh Q 17(3):288-307. https://doi.org/10. 1016/j.leaqua.2006.02.007

Cabrales ÁL, Medina CC, Lavado AC, Cabrera RV (2007) Managing functional diversity, risk taking and incentives for teams to achieve radical innovations: functional diversity, risk taking, incentives for teams to achieve radical innovations. R\&D Manag 38(1):35-50. https://doi.org/10.1111/j.1467-9310.2007.00501.x

Cheong M, Yammarino FJ, Dionne SD, Spain SM, Tsai C-Y (2019) A review of the effectiveness of empowering leadership. Leadersh $\mathrm{Q}$ 30(1):34-58. https://doi.org/10.1016/j.leaqua.2018.08.005

Creswell JW (2013) Research design: qualitative, quantitative, and mixed methods approaches. SAGE

Crevani L, Lindgren M, Packendorff J (2007) Leadership, not leaders: On the study of leadership as practices and interactions. Scand J Manag 26(1):77-86. https://doi.org/10.1016/j.scaman.2009.12. 003

Currie G, Spyridonidis D (2019) Sharing leadership for diffusion of innovation in professionalized settings. Human Relat 72(7):1209_ 1233. https://doi.org/10.1177/0018726718796175

Dewett T (2007) Linking intrinsic motivation, risk taking, and employee creativity in an R\&D environment. R\&D Manag 37(3):197208. https://doi.org/10.1111/j.1467-9310.2007.00469.x

Eisenbeiß SA, Boerner S (2010) Transformational leadership and R\&D innovation: taking a curvilinear approach: transformational leadership and innovation. Creat Innov Manag 19(4):364-372. https:// doi.org/10.1111/j.1467-8691.2010.00563.x

Ensley MD, Hmieleski KM, Pearce CL (2006) The importance of vertical and shared leadership within new venture top management teams: implications for the performance of startups. Leadersh Q 17(3):217-231. https://doi.org/10.1016/j.leaqua.2006.02.002

Field A (2017) Discovering statistics using IBM SPSS statistics (5. Aufl.). SAGE Publications

Furtner M, Baldegger U (2016) Self-Leadership und Führung: Theorien, Modelle und praktische Umsetzung (2. Aufl.). Gabler Verlag. https://www.springer.com/de/book/9783658130442
Furtner MR, Baldegger U, Rauthmann JF (2013) Leading yourself and leading others: linking self-leadership to transformational, transactional, and laissez-faire leadership. Eur J Work Organ Psy 22(4):436-449. https://doi.org/10.1080/1359432X.2012. 665605

Gritzo L, Fusfeld A, Carpenter D (2017) Exploring the principles of R\&D leadership with award-winning R\&D Leaders: successful leadership depends on performance across eight key areas. Res Technol Manag 60(3):18-21. https://doi.org/10.1080/08956308. 2017.1300999

Gumusluoğlu L, Ilsev A (2009) Transformational leadership and organizational innovation: the roles of internal and external support for innovation. J Prod Innov Manag 26(3):264-277. https://doi. org/10.1111/j.1540-5885.2009.00657.x

Hair JF (ed) (2010) Multivariate data analysis: A global perspective. Pearson, London

Hemphill JF (2003) Interpreting the magnitudes of correlation coefficients. American Psychol 58(1):78-79

Hoch JE, Pearce CL, Welzel L (2010) Is the most effective team leadership shared?: the impact of shared leadership, age diversity, and coordination on team performance. J Pers Psychol 9(3):105-116. https://doi.org/10.1027/1866-5888/a000020

Hoegl M, Parboteeah P (2006) Autonomy and teamwork in innovative projects. Hum Resour Manage 45(1):67-79. https://doi.org/10. 1002/hrm.20092

Hogan R, Kaiser RB (2005) What we know about Leadership. Rev Gen Psychol 9(2):169-180. https://doi.org/10.1037/1089-2680.9.2.169

Howell JM, Avolio BJ (1993) Transformational leadership, transactional leadership, locus of control, and support for innovation: key predictors of consolidated-business-unit performance. J Appl Psychol 78(6):891-902. https://doi.org/10.1037/0021-9010.78.6.891

Hughes DJ, Lee A, Tian AW, Newman A, Legood A (2018) Leadership, creativity, and innovation: a critical review and practical recommendations. Leadersh Q 29(5):549-569. https://doi.org/10. 1016/j.leaqua.2018.03.001

Ishikawa J (2012) Transformational leadership and gatekeeping leadership: the roles of norm for maintaining consensus and shared leadership in team performance. Asia Pac J Manag 29(2):265283. https://doi.org/10.1007/s10490-012-9282-z

Jung D, Wu A, Chow CW (2008) Towards understanding the direct and indirect effects of CEOs' transformational leadership on firm innovation. Leadersh Q 19(5):582-594. https://doi.org/10.1016/j. leaqua.2008.07.007

Junquera B, del Brío JÁ (2017) Is the leadership style important in R\&D departments in Spanish industrial companies? an empirical analysis. Technol Anal Strat Manag 29(10):1139-1152. https:// doi.org/10.1080/09537325.2016.1274024

Kearney E, Gebert D (2009) Managing diversity and enhancing team outcomes: the promise of transformational leadership. J Appl Psychol 94(1):77-89. https://doi.org/10.1037/a0013077

Keller RT (1992) Transformational leadership and the performance of research and development project groups. J Manag 18(3):489_ 501. https://doi.org/10.1177/014920639201800304

Keller RT (2006) Transformational leadership, initiating structure, and substitutes for leadership: a longitudinal study of research and development project team performance. J Appl Psychol 91(1):202-210. https://doi.org/10.1037/0021-9010.91.1.202

Kirkman BL, Harris TB (2017) 3-D team leadership: a new approach for complex teams. Stanford Business Books, an imprint of Stanford University Press

Kissi J, Dainty A, Tuuli M (2013) Examining the role of transformational leadership of portfolio managers in project performance. Int J Project Manage 31(4):485-497. https://doi.org/10.1016/j.ijpro man.2012.09.004

Lee A, Legood A, Hughes D, Tian AW, Newman A, Knight C (2019) Leadership, creativity and innovation: a meta-analytic review. Eur 
J Work Organ Psy 29(1):1-35. https://doi.org/10.1080/1359432X. 2019.1661837

Leech NL, Onwuegbuzie AJ (2009) A typology of mixed methods research designs. Qual Quant 43(2):265-275. https://doi.org/10. 1007/s11135-007-9105-3

Liu J, Liu X, Zeng X (2011) Does transactional leadership count for team innovativeness?: the moderating role of emotional labor and the mediating role of team efficacy. J Organ Chang Manag 24(3):282298. https://doi.org/10.1108/09534811111132695

Lovelace K, Shapiro DL, Weingart LR (2001) Maximizing cross-functional new product teams' innovativeness and constraint adherence: a conflict communications perspective. Acad Manag J 44(4):779-793. https://doi.org/10.2307/3069415

Mallick DN, Schroeder RG (2005) An integrated framework for measuring product development performance in high technology industries. Prod Oper Manag 14(2):142-158. https://doi.org/10.1111/j. 1937-5956.2005.tb00015.x

Nicolaides VC, LaPort KA, Chen TR, Tomassetti AJ, Weis EJ, Zaccaro SJ, Cortina JM (2014) The shared leadership of teams: a meta-analysis of proximal, distal, and moderating relationships. Leadersh Q 25(5):923-942. https://doi.org/10.1016/j.leaqua.2014.06.006

Nunnally JC (1978) An overview of psychological measurement. In: Wolman BB (ed) Clinical diagnosis of mental disorders. Springer, New York, pp 97-146. https://doi.org/10.1007/978-1-4684-2490-4_4

Paulsen N, Callan VJ, Ayoko O, Saunders D (2013) Transformational leadership and innovation in an R\&D organization experiencing major change. J Organ Chang Manag 26(3):595-610. https://doi. org/10.1108/09534811311328597

Peltokorpi V, Hasu M (2014) How participative safety matters more in team innovation as team size increases. J Bus Psychol 29(1):37-45. https://doi.org/10.1007/s10869-013-9301-1

Podsakoff PM, MacKenzie SB, Lee J-Y, Podsakoff NP (2003) Common method biases in behavioral research: a critical review of the literature and recommended remedies. J Appl Psychol 88(5):879-903. https://doi.org/10.1037/0021-9010.88.5.879

Rao Jada U, Mukhopadhyay S, Titiyal R (2019) Empowering leadership and innovative work behavior: a moderated mediation examination. J Knowl Manag 23(5):915-930. https://doi.org/10.1108/ JKM-08-2018-0533

Reagans R, Zuckerman E, McEvily B (2004) How to make the team: social networks vs. demography as criteria for designing effective teams. Adm Sci Q 49(1):101-133 (JSTOR)

Ryan RC, Tipu SAA (2013) Leadership effects on innovation propensity: a two-factor full range leadership model. J Bus Res 66(10):2116-2129

Rycroft RW (2007) Does cooperation absorb complexity? Innovation networks and the speed and spread of complex technological innovation. Technol Forecast Soc Chang 74(5):565-578. https://doi.org/ 10.1016/j.techfore.2006.10.005

Shin SJ, Zhou J (2007) When is educational specialization heterogeneity related to creativity in research and development teams? Transformational leadership as a moderator. J Appl Psychol 92(6):1709_ 1721. https://doi.org/10.1037/0021-9010.92.6.1709

Shirahada K, Niwa K (2007) Future-oriented mindset's contribution to management of corporate R\&D personnel motivation in Japan. Int J Innov Technol Manag 04(04):375-392. https://doi.org/10.1142/ S0219877007001144

Sims HP, Faraj S, Yun S (2009) When should a leader be directive or empowering? How to develop your own situational theory of leadership. Bus Horiz 52(2):149-158. https://doi.org/10.1016/j.bushor. 2008.10.002

Somech A (2006) The effects of leadership style and team process on performance and innovation in functionally heterogeneous teams. J Manag 32(1):132-157. https://doi.org/10.1177/0149206305277799
Srivastava A, Bartol KM, Locke EA (2006) Empowering leadership in management teams: effects on knowledge sharing, efficacy, and performance. Acad Manag J 49(6):1239-1251. https://doi.org/10.5465/ amj.2006.23478718

Stoker JI, Looise JC, Fisscher OAM, Jong RDD (2001) Leadership and innovation: Relations between leadership, individual characteristics and the functioning of R\&D teams. Int J Human Resour Manag 12(7):1141-1151. https://doi.org/10.1080/09585190110068359

Tang G, Chen Y, Knippenberg D, Yu B (2020) Antecedents and consequences of empowering leadership: leader power distance, leader perception of team capability, and team innovation. J Organ Behav 41(6):551-566. https://doi.org/10.1002/job.2449

Tekleab AG, Sims HP, Yun S, Tesluk PE, Cox J (2008) Are we on the same page? Effects of self-awareness of empowering and transformational leadership. J Leadersh Organ Stud 14(3):185-201. https:// doi.org/10.1177/1071791907311069

Vaccaro IG, Jansen JJP, Van Den Bosch FAJ, Volberda HW (2012) Management innovation and leadership: the moderating role of organizational size: management innovation and leadership. J Manage Stud 49(1):28-51. https://doi.org/10.1111/j.1467-6486. 2010.00976.x

Vecchio RP, Justin JE, Pearce CL (2010) Empowering leadership: an examination of mediating mechanisms within a hierarchical structure. Leadersh Q 21(3):530-542. https://doi.org/10.1016/j.leaqua. 2010.03.014

Waldman DA, Atwater LE (1994) The nature of effective leadership and championing processes at different levels in a R\&D hierarchy. J High Technol Manag Res 5(2):233-245. https://doi.org/10.1016/ 1047-8310(94)90004-3

Wang G, Oh I-S, Courtright SH, Colbert AE (2011) Transformational leadership and performance across criteria and levels: a meta-analytic review of 25 years of research. Group Org Manag 36(2):223-270. https://doi.org/10.1177/1059601111401017

Williamson JM, Lounsbury JW, Han LD (2013) Key personality traits of engineers for innovation and technology development. J Eng Tech Manage 30(2):157-168. https://doi.org/10.1016/j.jengtecman.2013. 01.003

Wong SI, Giessner SR (2018) The thin line between empowering and laissez-faire leadership: an expectancy-match perspective. J Manag 44(2):757-783. https://doi.org/10.1177/0149206315574597

Xue Y, Bradley J, Liang H (2011) Team climate, empowering leadership, and knowledge sharing. J Knowl Manag 15(2):299-312. https://doi. org/10.1108/13673271111119709

Yukl GA, Gardner WL (2020) Leadership in organizations (9. Aufl.). Pearson, London

Zhang X, Bartol KM (2010) Linking empowering leadership and employee creativity: the influence of psychological empowerment, intrinsic motivation, and creative process engagement. Acad Manag J 53(1):107-128

Zhu Y-Q, Chen H-G (2015) Empowering leadership in R\&D teams: a closer look at its components, process, and outcomes: Empowering leadership in R\&D teams. R\&D Manag 46(4):726-735. https://doi. org/10.1111/radm.12114

Zhu J, Yao J, Zhang L (2019) Linking empowering leadership to innovative behavior in professional learning communities: the role of psychological empowerment and team psychological safety. Asia Pac Educ Rev 20(4):657-671. https://doi.org/10.1007/ s12564-019-09584-2

Zibarras LD, Port RL, Woods SA (2008) Innovation and the 'Dark Side' of personality: dysfunctional traits and their relation to self-reported innovative characteristics. J Creat Behav 42(3):201-215. https://doi. org/10.1002/j.2162-6057.2008.tb01295.x

Zuraik A, Kelly L (2019) The role of CEO transformational leadership and innovation climate in exploration and exploitation. Eur J Innov Manag 22(1):84-104. https://doi.org/10.1108/EJIM-10-2017-0142 